\title{
Class-Controlled Copy-Paste Based Cell Segmentation for CoNIC Challenge
}

\author{
Heeyoung Ahn \\ Department of R\&D Center, \\ Arontier Co., Ltd \\ Seoul, Republic of Korea
}

\author{
Yiyu Hong \\ Department of R\&D Center, \\ Arontier Co., Ltd \\ Seoul, Republic of Korea
}

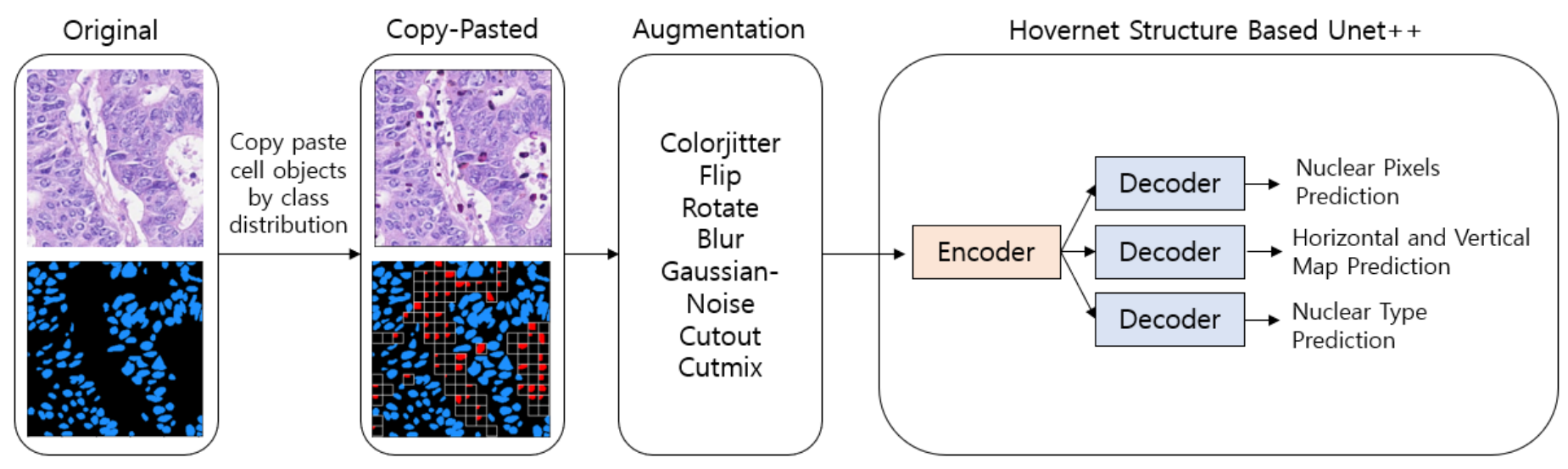

Fig1. Overall Architecture of our proposed method.

\begin{abstract}
Muti-class cell segmentation in histopathology images is a challenging task. Here, we propose a copy-paste augmentation-based method for CoNIC challenge. As the challenge train data is severely class imbalanced. To deal with it, we copy all cell objects of train data and paste them to the train image on the fly while training model. The paste strategy is that we paste more cell objects of the insufficient classes and paste less cell objects for the sufficient classes. We experimented the method by stratified splitting train data in 4:1 ratio, the result shows the copy paste method can reach PQ 64.84 and $\mathrm{mPQ}$ 53.72, which improved 0.6 and 0.66 compared to without copy pasted. Moreover, the improvements in those insufficient classes is more obvious.
\end{abstract}

\section{INTRODUCTION}

This manuscript describes submitted method of team Arontier to CoNIC Challenge [1-2]. Our method mainly focus on how to deal with the class imbalance using copy-paste augmentation[1] with a little bit modification. We increase the number of cell objects for insufficient classes (Neutrophil, Plasma, Eosinophil) intensively and for those sufficient classes weakly using copy-paste augmentation, respectively. Importantly, considering there is no improvements when pasting cells to where they would be overlapped with other originally existed cells, we paste the cell objects to where they would not be overlapped.
Unlike copy-paste augmentation[3] which do copy-paste in 2 different image pair, we want to paste cells from any images to target image. We extract all cell objects from all training set and saved them in a file in advance. As a result, we can efficiently use these cell objects and paste them on target train images when training our model on the fly.

\section{B. Source of pasted cells}

For our copy-paste augmentation, we consider two method. One is source-level and the other is random-level. In source level, considering there are five image sources(i.e., consep, crag, dpath, glas, pannuke) in datasets, we paste cells from image of specific source to other target image of same source. For example, we extract cells from images of consep source and paste those cells to target images of same consep source. Unlike this, in randomlevel we extract cells from images of all sources and paste those cells to target images randomly without considering any sources. In our experiments, we found that random level yields better performance over source-level.

\section{Calculate candidate box region}

For pasting cells to none-overlapping region, we firstly calculate the empty candidate box region to which the cells would be pasted. To this end, we stride a window in target image and calculate the total number of candidate box region to which cells would be pasted. Considering various size of cells, we select window size with 15-pixel width and height.

\section{METHOD}


After calculating the total number of candidate box region in each image, we set the ratio of how many boxes to use. We select random values of 0.1 interval between 0.1 and 1 . Then we as sign different ratio for each class according to the number of cells of a particular class in training images. Specifically, we assign different ratio of $0.3,0.1,0.05,0.15,0.3,0.1$ for each class because we want to deal with the insufficiency of particular classes. Finally if the pasted cells still overlap with other cells, we skip those cells.

\section{E. Augmentation for pasted cell}

We experimented several augmentation method such as flip, scaling for cells which will be pasted. We found that scaling was not effective so we just use horizontal, vertical flip.

\section{TRAINING DETAILS}

We follow the HoverNet[2] but with some differences. We replace the HoverNet[2] model structure with Unet++[3] and modify the structure so that model can output 3 outputs like HoverNet[2]. We select EfficientNet-b7[4] pretrained on ImageNet[5] for encoder of Unet++[3]. For Image Augmentation, we choose ColorJitter, Flip, Rotate, Blur, Gaussian Noise, Cutout [6], Cutmix[7]. We select Radam [10] optimizer with learning rate of 1e-3. During training, we downscale learning rate from 1e-3 to 1e-5 using step scheduler with 0.1 ratio every 100 epoch. For loss function, we choose same loss function that baseline of challenge [1] applied except for classification output. For classification output, we choose focal loss [11] with gamma 2, alpha 1 . We train the model with batch size 8 for 300 epochs. Finally, we ensemble outputs of 5 models which were trained in 5-Fold cross validation setting.

\section{RESULTS}

TABLE 1. Results of model with / without proposed copy-paste for total classes on CoNIC challenge Dataset.

\begin{tabular}{|l|ll|}
\hline Model & PQ & mPQ \\
\hline Unet++ & 64.24 & 53.06 \\
w/ Copy-Paste & $64.84(\uparrow 0.6)$ & $53.72(\uparrow 0.66)$ \\
\hline
\end{tabular}

TABLE 2. Results of model with / without proposed copy-paste for each class on CoNIC challenge Dataset.

\begin{tabular}{|l|ll|}
\hline Classes & Model & PQ \\
\hline 1. Neutrophil & Unet++ & 31.32 \\
& w/ Copy-Paste & $31.99(\uparrow 0.67)$ \\
2. Epithelial & Unet++ & 63.49 \\
& w/ Copy-Paste & $63.81(\uparrow 0.32)$ \\
3. Lymphocyte & Unet++ & 72.90 \\
& w/ Copy-Paste & $73.03(\uparrow 0.13)$ \\
4. Plasma & Unet++ & 52.39 \\
& w/ Copy-Paste & $53.47(\uparrow 1.08)$ \\
5. Eosinophil & Unet++ & 38.18 \\
& w/ Copy-Paste & $39.80(\uparrow 1.62)$ \\
6. Connective & Unet++ & 60.04 \\
& w/ Copy-Paste & $60.18(\uparrow 0.14)$ \\
\hline
\end{tabular}

TABLE 3. Results of model for submission.

\begin{tabular}{|l|ll|}
\hline Fold & PQ & mPQ \\
\hline Fold1 & 64.91 & 53.49 \\
Fold2 & 65.35 & 54.01 \\
Fold3 & 65.65 & 54.45 \\
Fold4 & 65.09 & 54.98 \\
Fold5 & 65.77 & 53.95 \\
\hline
\end{tabular}

For validating proposed copy-paste augmentation, we split datasets stratifiedly by $4: 1$ (train : valid) setting which is same as baseline of challenge. Table 1, 2 shows the results of applying proposed copy-paste augmentation for total classes and each class, respectively. When applied proposed copy-paste augmentation, for total classes PQ and mPQ improve by 0.6 , 0.66 respectively and for each class PQ improve by $0.67,0.32$, $0.13,1.08,1.62,0.14$ respectively on validation set. Finally, Table 3 shows 5-Fold cross validation results of our model for submission.

\section{REFERENCE}

[1] Graham, Simon, et al. "CoNIC: Colon Nuclei Identification and Counting Challenge 2022." arXiv preprint arXiv:2111.14485 (2021).

[2] Graham, Simon, et al. "Lizard: A Large-Scale Dataset for Colonic Nuclear Instance Segmentation and Classification." Proceedings of the IEEE/CVF International Conference on Computer Vision. 2021.

[3] Golnaz Ghiasi, Yin Cui, Aravind Srinivas, Rui Qian, Tsung-Yi Lin, Ekin D. Cubuk, Quoc V. Le, Barret Zoph. Simple Copy-Paste is a Strong Data Augmentation Method for Instance Segmentation. In CVPR, 2021.

[4] Simon Graham, Quoc Dang Vu, Shan E Ahmed Raza, Ayesha Azam, Yee Wah Tsang, Jin Tae Kwak, Nasir Rajpoot. HoVer-Net: Simultaneous Segmentation and Classification of Nuclei in MultiTissue Histology Images. In Medical Image Analysis, 2018.

[5] Zongwei Zhou, Md Mahfuzur Rahman Siddiquee, Nima Tajbakhsh, Jianming Liang. UNet++: A Nested U-Net Architecture for Medical Image Segmentation. In CVPR, 2018.

[6] Mingxing Tan, Quoc V. Le. EfficientNet: Rethinking Model Scaling for Convolutional Neural Networks. In ICML, 2019.

[7] Olga Russakovsky, Jia Deng, Hao Su, Jonathan Krause, Sanjeev Satheesh, Sean Ma, Zhiheng Huang, Andrej Karpathy, Aditya Khosla, Michael Bernstein, et al. Imagenet large scale visual recognition challenge. In IJCV, 2015.

[8] Terrance DeVries, Graham W. Taylor. Improved Regularization of Convolutional Neural Networks with Cutout. In CVPR, 2017.

[9] Sangdoo Yun, Dongyoon Han, Seong Joon Oh, Sanghyuk Chun, Junsuk Choe, Youngjoon Yoo. CutMix: Regularization Strategy to Train Strong Classifiers with Localizable Features. In CVPR, 2019.

[10] Liyuan Liu, Haoming Jiang, Pengcheng He, Weizhu Chen, Xiaodong Liu, Jianfeng Gao, Jiawei Han. On the Variance of the Adaptive Learning Rate and Beyond. In ICLR, 2019.

[11] Tsung-Yi Lin, Priya Goyal, Ross Girshick, Kaiming He, Piotr Dollár. Focal Loss for Dense Object Detection. In CVPR, 20 
bioRxiv preprint doi: https://doi.org/10.1101/2022.03.02.482203; this version posted March 4, 2022. The copyright holder for this preprint (which was not certified by peer review) is the author/funder, who has granted bioRxiv a license to display the preprint in perpetuity. It is made available under aCC-BY-NC 4.0 International license. 